1 Living Well GP Partnership, Southampton, UK

2 Primary Medical Care, School of Primary Care, Population Sciences and Medical Education, University of Southampton, UK

3 University Hospital Southampton NHS Foundation Trust

4 University of Southampton, UK

Correspondence to: Pritti Aggarwal Prittiaggarwal@nhs.net

Cite this as: BMJ 2021;375:n3005 http://dx.doi.org/10.1136/bmj.n3005 Published: 14 December 2021
CHRISTMAS 2021: TRADING PLACES

\section{Gatekeepers, wizards, and a mutual appreciation of each other's kingdoms}

\author{
Pritti Aggarwal, ${ }^{1,2}$ Harnish P Patel 3,4
}

Collaborative working is crucial to providing joined up, high quality care, yet medicine continues to be a divided profession of gatekeepers (GPs) and wizards (specialists). ${ }^{12}$ The covid-19 pandemic has exacerbated this sense of estrangement, with ever increasing workloads not conducive to goodwill across healthcare teams.

More than ever, we need to create a shared understanding of each other's roles and ignite opportunities for interprofessional education and communication. We both participated in a GP-consultant exchange scheme just before the pandemic ${ }^{3}$ and share how it's shaped our perception of each other's work and our practice.

\section{Insights into a wizard's kingdom from a gatekeeper}

The last time I stepped into a hospital department was when I was training, a good 15 years ago. I was shocked by how bustling it was. Everyone-porters, cleaners, catering staff, therapists, clinical teams, allied health professionals, nurses-was vying for space on the ward.

I was impressed by the stamina my hospital colleagues needed to sustain a three to four hour ward round while leading, teaching, instructing junior teams, and interacting with members of the multidisciplinary team to make complex decisions about patient care. Their momentum, enthusiasm, and professionalism were inspiring to see. By the end of the day in practice I'm often tired from decision making-but this was a whole new level of fatigue.

Invariably, after the ward round, my colleagues then switched to a new activity-including outpatient clinics, rehabilitation rounds, theatre sessions, teaching, training, or developing their own specialist skills. I gained a newfound admiration for how many plates specialists spin simultaneously.

I learnt that the referral pathway is highly complex; there are over 21 steps from my referral to a procedure. Observing the multiple hospital IT systems at work-that didn't always link with each other, or with primary care-gave me an appreciation of how lucky I am to have one comprehensive IT system in my GP surgery. After seeing this-and knowing that I have access to a patient's whole medical record-I now write more detailed referral letters to support my secondary care colleagues. This has become even more important during the pandemic as many patient journeys are more fragmented, with multiple agency contacts all under the umbrella of the NHS-for example, 111, NHS Test and Trace, emergency departments, out of hours services, and hot clinics.

More than anything, though, I saw highly skilled and compassionate colleagues spending a significant amount of time with patients-an experience that boosted my morale and which has continued to sustain me through difficult times in primary care. I now better appreciate our differing specialist skills. I am a true generalist, recognising the boundaries of my skill, but I am fortunate to have a vast secondary care resource at my doorstep, supporting me to take care of my patients should the need arise.

\section{Insights into a gatekeeper's kingdom from a wizard}

I was struck by the wealth of knowledge GPs have and how they use all their faculties to diagnose, manage, and safety net a range of conditions of varying complexity. As someone who can take an hour or more to complete a geriatric assessment, I found seeing these principles followed in a 10 minute consultation a sight to behold.

I was slightly taken aback at how isolated GPs were in practice: desk, screen, and couch-their second home. I was amazed at the camaraderie they shared in spite of this, but am still grateful for the interactive environment I have in hospital.

It's easy for narratives of blame and of work being "dumped" on each other to arise between primary and secondary care, but all I saw was a genuine respect for, and efforts to support, secondary care, with GPs keen to avoid hospital admissions and outpatient referrals. I was humbled by how much clinical uncertainty GPs live with as a consequence of their limited access to investigations and the absence of or delay in communication from secondary care in the form of outpatient letters or discharge summaries.

I was impressed by how much information about a patient's baseline function and social circumstances GPs know or have in their electronic health records, but this is often not sought in secondary care.

Equally, I've come to see how the information we give about the patient through our discharge summaries is not always conducive to effective patient management. On a ward round, I'll often bat out comments before discharging a patient, such as "get GP to check U\&E in one week," without giving much thought to the reasons or its implications. These offhand requests now seem ridiculous, knowing that my discharge summary is among hundreds of correspondence letters to hit the practice that day, 
each specifying delegated tasks that together contribute to an unmanageable GP workload. Seeing primary care colleagues at work has made me rethink my overall responsibility for organising suitable follow-up of these types of investigations. I don't hesitate now to pick up the phone to contact my GP colleagues to facilitate seamless transfer of care. Getting to hear a voice on the line, and knowing there's a person at the other end of it, has been even more important during the pandemic.

\section{Creating a single kingdom}

Wizards and gatekeepers need each other, but this recognition of our mutual dependence can easily become lost, especially amid the stresses and demands of the past two years. We were able to use a local GP-consultant exchange scheme to gain an insight into each other's working day, but embedding this kind of learning into the curriculums of all trainees, as well as within the core values of trusts and integrated care systems, would help to ensure that workload does not become a barrier to other doctors gaining these experiences. 45

Collaboration starts with us all recognising the arbitrary primary-secondary care divide for what it is. If we want to recover from this pandemic and deliver an NHS fit for the 21st century, then neither side can do it alone: we'll need to work together as a single kingdom. ${ }^{6}$

Competing interests: PA is a gatekeeper married to a wizard. HPP is a wizard married to a gatekeeper.

1 Herd B, Herd A, Mathers N. The wizard and the gatekeeper: of castles and contracts. BMJ 1995;310:1042-4. doi: 10.1136/bmj.310.6986.1042 pmid: 7728059

2 Mathers N, Hodgkin P. The Gatekeeper and the Wizard: a fairy tale. BMJ1989;298:172-4. doi: 10.1136/bmj.298.6667.172 pmid: 2493843

3 NHS England. Wessex Model for workplace exchanges: learning and next steps from the consultant GP exchange, Southampton 2018. 1 December 2019. www.england.nhs.uk/publication/the-wessex-model-for-workplace-exchanges-learning-and-next-steps-from-the-consultantgp-exchange-southampton-2018.

4 Sampson R, Barbour R, Wilson P. The relationship between GPs and hospital consultants and the implications for patient care: a qualitative study. BMC Fam Pract 2016;17:45. doi: 10.1186/s12875-016-0442-y pmid: 27074867

5 Sampson R, MacVicar R, Wilson P. Development of an interface-focused educational complex intervention. Educ Prim Care 2017;28:265-73. doi: 10.1080/14739879.2017.1309690 pmid: 28394242

6 Cox J. GPS can no longer claim to be the "gatekeepers" of the NHS. Br J Gen Pract 2006;56:83-4.pmid: 16464318

7 BGS voices support for primary care following criticism. British Geriatrics Society. 15 September 2021. www.bgs.org.uk/policy-and-media/bgs-voices-support-for-primary-care. 